\title{
Contexto, Desafios e Perspectivas na Formação de Obstetrizes no Brasil
}

\section{Midwifery Training in Brazil: Context, Challenges and Perspectives}

\author{
Nádia Zanon Narchi \\ Livre-docente em Saúde Materna e Perinatal. Coordenadora e \\ Docente do Curso de Obstetrícia. Escola de Artes, Ciências e Hu- \\ manidades da Universidade de São Paulo. \\ Endereço: Avenida Arlindo Bettio, 1000, Ermelino Matarazzo, CEP \\ 03828-000, São Paulo, SP, Brasil. \\ E-mail: nznœusp.br \\ Lúcia Cristina Florentino Pereira da Silva \\ Doutora em Enfermagem. Docente do Curso de Obstetrícia. Escola \\ de Artes, Ciências e Humanidades da Universidade de São Paulo. \\ Endereço: Avenida Arlindo Bettio, 1000, Ermelino Matarazzo, CEP \\ 03828-000, São Paulo, SP, Brasil. \\ E-mail: lucrisळusp.br \\ Dulce Maria Rosa Gualda \\ Profa Titular do Departamento de Enfermagem Materno-infantil \\ e Psiquiátrica da Escola de Enfermagem da Universidade de São \\ Paulo. \\ Endereço: Avenida Arlindo Bettio, 1000, Ermelino Matarazzo, CEP \\ 03828-000, São Paulo, SP, Brasil. \\ E-mail: drgualdaœusp.br
}

\section{Resumo}

O artigo descreve o processo de criação do Curso de Obstetrícia da Escola de Artes, Ciências e Humanidades da Universidade de São Paulo. Ele apresenta o projeto político pedagógico e os referenciais teóricos que suportam a formação de obstetrizes, a resistência ou os movimentos que se opõem à formação e ao registro desses profissionais, os desafios que precisam ser superados e, finalmente, as perspectivas nas quais as obstetrizes podem contribuir não só para melhorar a qualidade dos cuidados em saúde, como também para diminuir as taxas de morbidade e mortalidade materna e perinatal e de cesarianas desnecessárias no país.

Palavras-chave: Obstetriz; Educação superior; Prática profissional. 


\section{Abstract}

This article describes the creation process of the first direct-entry Midwifery Course in Brazil, at the School of Arts, Sciences and Humanities of the University of São Paulo, Brazil. It presents the course's political-educational project and the theoretical references that support it; the movements or resistances that are opposed to the training and registration of midwives; the challenges that need to be overcome. Finally, the paper presents the foreseen perspectives of contribution of these professionals towards the improvement of healthcare quality, the decrease in maternal and perinatal morbidity and mortality rates, as well as of unnecessary cesarean sections in the country.

Keywords: Midwife; Education, Higher; Professional Practice.

\section{Contexto em que ocorre o Curso de Obstetrícia}

Em 2005 a Universidade de São Paulo (USP) criou a Escola de Artes, Ciências e Humanidades (EACH) como uma unidade integrada. Aí foram criados dez cursos que ainda não eram oferecidos em outras unidades da instituição, entre eles o de Graduação em Obstetrícia.

A EACH representa proposta inovadora que tem por base a interdisciplinaridade como ferramenta para a construção de conhecimentos e rompe com o modelo tradicional dos departamentos da USP. Seu modelo de ensino baseia-se no diálogo constante entre as diferentes áreas de saber e busca oferecer formação acadêmica ampla, que possibilite aos futuros profissionais uma leitura crítica do mundo, bem como a excelência nas suas áreas específicas.

A constituição da EACH baseou-se também em forte compromisso com o atendimento das demandas da comunidade da Zona Leste do município de São Paulo, o que exige, além do ensino e pesquisa, trabalhos articulados de extensão que visem promover melhorias no contexto local.

A operacionalização do modelo EACH prevê que os alunos cursem disciplinas de um Ciclo Básico (CB) comum no primeiro ano de todos os cursos de graduação. O CB foi idealizado para promover tanto a iniciação acadêmica e discussão interdisciplinar no campo das ciências, das artes e das humanidades quanto a articulação do conhecimento com a realidade da sociedade brasileira, regional e local. Ele se estrutura em três eixos centrais:

1. Formação introdutória no campo específico de conhecimentos de cada curso: leva os estudantes a tomarem contato com as bases conceituais de seu curso, permitindo que comecem a conhecer o campo profissional que escolheram no vestibular.

2. Formação geral: aponta a complexidade dos fenômenos naturais, sociais e culturais, oferecendo formação integrada nas áreas de ciências naturais, de humanidades e de artes, com ênfase em aspectos teóricos e metodológicos, fundamentados nas bases filosóficas do conhecimento científico, das relações sociedade-natureza, dos aspectos socioculturais da sociedade contemporânea, com noções sobre direitos humanos, cidadania e outros mais. 
3. Formação científica por meio da Resolução de Problemas (RP): promove e incentiva a iniciação e formação científica dos alunos, articulando-se com os eixos anteriormente citados.

Os princípios e os objetivos do CB comprometemse com uma formação de caráter científico, social e político que permitem a diferenciação profissional. Nessa perspectiva, a RP é um dos eixos centrais da proposta pedagógica da EACH, que adota como princípio o papel ativo dos estudantes na construção do conhecimento e se inspira nos movimentos internacionais que organizam os currículos a partir do PBL (Problem-Based Learning), metodologia que se caracteriza pelo uso de problemas da vida real para estimular o pensamento crítico e o aprendizado de conceitos relacionados à área específica de conhecimento e que, especificamente na área da saúde, tem sido apontada como uma das mais promissoras inovações no campo da educação, graças aos resultados que propicia e à coerência entre os seus fundamentos e as principais teorias de aprendizagem de adultos (Barret, 2001; Barrows, 1996).

O projeto acadêmico do CB da EACH procura garantir a formação disciplinar específica, geral e científica por meio da RP, articulando-se com experiências internacionais, cujos resultados demonstram boa qualidade acadêmica dos estudantes e conexão com as necessidades sociais das empresas e entidades públicas.

Por estes motivos, diversos cursos da EACH, entre eles o de Obstetrícia, adotam a metodologia de RP em outros semestres e disciplinas, a partir do reconhecimento do caráter inovador e potencializador desta metodologia no processo de aprendizagem na área da saúde (McCourt e Thomas, 2001; Rowan e col., 2009).

\section{O Contexto Histórico da Criação do Curso de Obstetrícia e suas Referências Ético-Legais}

Desde a década de oitenta, a assistência à saúde da mulher no Brasil tem sido objeto de muitas discussões e de várias tentativas de mudanças no âmbito das políticas públicas de saúde. Pode-se citar como exemplo a criação de programas, resoluções e leis que visam à humanização do parto e nascimento, a redução do número de cesarianas e dos índices de morbi-mortalidade materna e perinatal (Brasil, 2000, 2001, 2004, 2008).

Estes temas tornaram-se pauta dos movimentos sociais de saúde e de mulheres que reivindicam o pleno exercício dos direitos sexuais e reprodutivos e ressaltam o direito de escolha do tipo de parto e da autonomia da mulher. Nesse processo, maternidades públicas e privadas têm buscado implementar mudanças no modelo de cuidado prestado à mulher, ao recém-nascido e à sua família, o que necessariamente inclui alterações estruturais, funcionais e de recursos humanos. A criação de Centros de Parto Normal intra-hospitalares, por exemplo, advém dessa mudança conceitual e fundamenta-se tanto na assistência humanizada, com base em evidências científicas, quanto na prestação direta de cuidado pelas enfermeiras obstetras, profissionais reconhecidamente capacitadas para promover melhoria da qualidade assistencial e, consequentemente, maternidade mais segura (Brasil, 2001; Calderon e col., 2006; Cecatti e Calderon, 2005; Costa e col., 2005; Di Mario e col., 2005; Hatem e col., 2008; Lansky e col., 2002).

No entanto, essas estratégias têm sido insuficientes para reduzir as taxas de mortalidade materna e perinatal, cujos elevados índices se mantém estabilizados desde o ano de 2001. Isto é devido a fatores socioeconômicos e culturais das mulheres brasileiras, bem como à inadequada qualidade da assistência materna e perinatal, de forma especial a dos serviços públicos de saúde (Rattner, 2009a, 2009b; Brasil, 2010).

Além disso, a despeito das evidências científicas e das recomendações de organizações nacionais e internacionais, o Brasil apresenta o mais elevado índice de cesarianas da América Latina: 40\% no serviço público e $85 \%$ no setor privado em 2006, o que também tem contribuído para manter elevados os índices de morbi-mortalidade materna e perinatal e levou o governo brasileiro a estabelecer novas estratégias para promover o parto normal e reduzir as cesáreas desnecessárias (Brasil, 2008).

No entanto, outra estratégia considerada essencial para a redução de tais índices se relaciona com o aumento quantitativo e com o incremento na 
qualificação dos profissionais que assistem a mulher durante todo o ciclo gravídico-puerperal (WHO, 2001, 2004). Com base nessas necessidades é que surgiu a ideia de retomar a formação direta de obstetrizes de acordo com as diretrizes definidas pela Confederação Internacional das Obstetrizes (ICM) e pela Organização Mundial da Saúde (OMS) para a promoção da maternidade segura e para a formação de parteiras profissionais (WHO, 2001, 2004; 2006). Este modelo já existia no Brasil e na própria USP até 1970 e ainda prevalece em países como Inglaterra, Holanda, Estados Unidos, Chile, entre outros.

A formação de obstetrizes não exclui as enfermeiras da atenção. Pelo contrário, pretende fortalecer um modelo de atenção que não seja centrado no modelo biomédico, ainda presente na formação geral de Enfermagem, ou apenas na assistência ao parto. Essa crítica é feita por muitas enfermeiras que, ao questionarem seu modelo de formação e, consequentemente, de trabalho, buscam transformar seu fazer. Nesse processo, elas encontram diversos obstáculos, em decorrência de o mercado ser ainda limitado, a despeito da legislação e de todo incentivo governamental que assegura e legitima a autonomia das enfermeiras obstetras, habilitadas ou especialistas, para assistir às mulheres durante a gestação, parto e pós-parto (Carvalho, 200o; Narchi, 2001).

A esse respeito observa-se que muitas enfermeiras obstetras se autodenominam obstetrizes, talvez por sua maior identificação com a "Midwifery" ou com a "Parteria", termos que infelizmente não encontram tradução na língua portuguesa. Para o ICM (2005), obstetrizes (midwives) são profissionais formadas por um programa regular de ensino, o que não se aplica às parteiras leigas ou tradicionais, chamadas popularmente de "curiosas", por terem sido "formadas" pela tradição e cultura no ofício da assistência às mulheres durante o parto.

A despeito de o substantivo obstetriz ser feminino, convencionou-se na USP que os homens formados pelo Curso de Obstetrícia também sejam assim nominados para que não haja confusão com o substantivo masculino equivalente, "obstetra”, denominação profissional culturalmente associada no Brasil à classe médica.

Conforme salientado, já houve a formação de parteiras profissionais ou obstetrizes no Brasil.
Os primeiros programas regulares iniciaram-se no ano de 1832, primeiro no Estado do Rio de Janeiro e depois na Bahia, Rio Grande do Sul, São Paulo e Pará (Jorge, 1975). Por este motivo, na década de 20 do século XX, profissionais organizadas fundaram a Associação Brasileira de Obstetrizes (ABO), entidade que, na década de 8o, inspirou a criação da Associação Brasileira de Obstetrizes e Enfermeiros Obstetras (ABENFO).

Por congregar obstetrizes e enfermeiros obstetras, a ABENFO sempre participou das discussões a respeito da reativação do Curso de Obstetrícia, cujo projeto foi amplamente discutido em eventos promovidos pela entidade, tais como os $3^{\circ}$ e $4^{\circ}$ Seminários Estaduais sobre o Ensino de Enfermagem para a Assistência ao Parto e Nascimento, de 2001 e 2004, e os $3^{\circ}$ e $4^{\circ}$ Congressos Brasileiros de Enfermagem Obstétrica e Neonatal, de 2002 e 2004.

Como o projeto pedagógico do curso está em processo de avaliação permanente desde sua implantação, ele já foi reestruturado e aprimorado a fim de corresponder às expectativas de docentes e discentes, de melhor atender as necessidades de saúde da população e de seguir as competências essenciais em obstetrícia, estabelecidas pelo ICM (2002) no intuito de aprimorar o ensino da Obstetrícia, auxiliar a regulação profissional, desenvolver padrões de prática e fortalecer a profissão. Neste caminho evolutivo, a ênfase nos aspectos fisiológicos, emocionais e sócio-culturais do processo reprodutivo e na transformação do modelo de atenção à maternidade permanece sendo o sustentáculo da formação de obstetrizes pela USP.

As referências legais no âmbito internacional para o curso são o Código Internacional de Ética para as Obstetrizes e as Competências Essenciais para a Prática da Obstetrícia, ambas definidas pelo ICM, confederação que congrega obstetrizes de 72 países diferentes e que trabalha em conjunto com a OMS, o Fundo das Nações Unidas para a Infância (UNICEF), a Organização dos Estados Americanos (OEA) e o Fundo das Nações Unidas para Populações (UNFPA). As ações do ICM estão organizadas no sentido de promover maternidade mais segura em todo o mundo e de estimular a formação de obstetrizes como uma profissão respeitada e atraente, regulamentada e com ingresso garantido no sistema de 
saúde (ICM, . 2002, 2005, 2011; OEA, 2006; UNFPA, 2006, 2007; WHO, 2001, 2004).

Por se compreender que obstetrizes façam parte das equipes de enfermagem, ou seja, que possam trabalhar de forma colaborativa e em parceria com enfermeiros, auxiliares e técnicos de enfermagem, como ainda hoje acontece com as formadas até o ano de 1970, considera-se que as referências legais nacionais para o Curso de Obstetrícia sejam o Código de Ética dos Profissionais de Enfermagem, a Lei do Exercício Profissional da Enfermagem, $n^{\circ} .7498$ de 1986, e o Decreto que a regulamenta, $\mathrm{n}^{\circ} .94 .406 \mathrm{de}$ 1987 (COREN-SP, 2009).

Como esta legislação prevê o trabalho de obstetrizes, limitando sua atuação à assistência ao parto, resta definir, com base tanto na realidade brasileira quanto nas competências profissionais específicas, de que forma ocorrerá a interface entre a Enfermagem e a Obstetrícia.

Levando em conta que o escopo de trabalho de enfermeiras obstetras e de obstetrizes não se limita única e exclusivamente à assistência ao parto, verifica-se como é possível a coexistência dessas duas profissões em um país com dimensões continentais e com realidades tão díspares como o Brasil. Nesse sentido, observa-se que a formação de obstetrizes inclui aspectos fundamentais, tais como o monitoramento do bem-estar físico, psicológico, espiritual e social das mulheres e famílias, bem como a continuidade de cuidado durante toda a gravidez, parto e pós-parto; a promoção de educação individualizada à mulher, o aconselhamento e o acompanhamento prénatal; o contínuo cuidado/suporte/atenção durante todas as fases do trabalho de parto e do pós-parto; a restrição ao uso de intervenções tecnológicas desnecessárias, entre outros aspectos apontados pelo ICM e pela OMS para a obtenção da maternidade segura (ICM, 2011, 2005; WHO, 2001, 2004).

\section{Marcos Conceituais e Organização Curricular do Curso de Obstetrícia}

Seguindo as definições do ICM (2005), o profissional formado pelo Curso de Obstetrícia da EACHUSP deve trabalhar em parceria com as mulheres fornecendo-lhes o necessário suporte, cuidado e aconselhamento durante a gravidez, parto e pós- parto. Essa atenção inclui medidas de prevenção de agravos, de promoção do parto normal, de condução de partos, de provisão de cuidados ao recém-nascido e ao lactente, de detecção de complicações na saúde materna e perinatal, de acesso ao cuidado médico ou a outro tipo de assistência apropriada e de realização de medidas de emergência. Além disso, a/o obstetriz tem uma tarefa importante na educação e na promoção da saúde, não só das mulheres, como também da família e da comunidade. Seu trabalho deve incluir a educação pré-natal e a preparação para a paternidade, estendendo-se à atenção integral à saúde da mulher e à assistência à criança até os dois meses de vida.

Como profissional autônomo ou trabalhando em equipes multiprofissionais, a/o obstetriz pode exercer seu trabalho em hospitais, consultórios, unidades de saúde, domicílios ou em qualquer outro tipo de serviço de saúde. Em relação ao alcance da prática profissional, o ICM (2005) esclarece que este deve ser idealmente conduzido dentro de um sistema de atenção à saúde baseado na comunidade, que pode incluir parteiras tradicionais, trabalhadores de saúde comunitária, médicos, enfermeiros e especialistas em centros de referência.

Por estas razões, o objetivo geral do Curso de Obstetrícia é o de formar um profissional apto a trabalhar na promoção integral da saúde no contexto do Sistema Único de Saúde (SUS) e das premissas da humanização do parto e do nascimento. Para isso, os graduados em Obstetrícia recebem uma formação geral voltada para o compromisso com a cidadania e a busca de soluções de problemas contemporâneos e uma formação específica que integra dimensões biológicas, psicossociais e culturais no processo de cuidado e assistência às mulheres, suas famílias e comunidade.

O curso prepara, dessa maneira, profissionais qualificados para prestar e coordenar a assistência à saúde da mulher e família no processo reprodutivo; atuar em equipe multiprofissional; desenvolver processo interativo com os diversos níveis de atuação em saúde; contribuir para a construção do conhecimento na área e fundamentar a sua prática naquele já existente; analisar criticamente a realidade de assistência à saúde da mulher, propondo ações criativas para a solução dos problemas encontra- 
dos, levando em conta o perfil epidemiológico, os fatores sócio-políticos e culturais, a tecnologia e os equipamentos disponíveis e necessários à prática profissional.

A fim de atingir formação integral do estudante, o projeto político pedagógico do curso, cuja carga horária total é de 4140 horas, tem como base os referenciais teóricos críticos-reflexivos, nos quais o processo de construção do conhecimento é necessariamente dialógico, diferentemente do que ocorre na maioria dos currículos dos cursos de graduação na área da saúde (Albuquerque e col., 2009). Nesse contexto, a integralidade, eixo norteador de novas formas de agir social em saúde, se faz presente em todas as dimensões da formação. Isto significa dizer que o curso busca formar profissionais capacitados para o cuidado integral em saúde e para trabalhar na promoção de saúde das pessoas, grupos e comunidade (Pinheiro e col., 2003).

Nas discussões e ações desenvolvidas em toda a formação em Obstetrícia estão presentes os pressupostos da Humanização do Cuidado em Saúde (Ayres, 2005; Maia, 2005), em especial aqueles relacionados ao ciclo gravídico-puerperal (Brasil, 2001) e às recomendações da Organização Mundial da Saúde (WHO, 2004). Estes preconizam o mínimo possível de intervenções na gestação, no parto e no puerpério, a importância das evidências científicas e dos direitos das mulheres à informação e à tomada de decisão sobre suas necessidades de saúde.

Como a prática da obstetrícia é compreendida como uma arte baseada em bom senso, tradição e cuidado centrado na pessoa e como a maioria das mulheres que buscam a atenção obstétrica é saudável e requer apenas cuidados básicos de promoção à saúde, o trabalho das obstetrizes é prestar assistência integrada, individualizada e baseada na melhor e mais atualizada evidência científica (Fullerton e Thompson, 2005).

Pode-se dizer, com base nesses referenciais, que do ponto de vista ético e político o curso busca articular três dimensões: a reflexão sobre a realidade social e a construção de conhecimentos, o compromisso social com o SUS e com o cuidado integral em saúde e, por fim, a adoção de modelo de cuidado centrado na mulher e o cumprimento das políticas de humanização do parto e do nascimento.
Com base nesses pressupostos, os quatro anos e meio do curso estão estruturados no CB, no primeiro ano, e em diversas disciplinas divididas em três eixos fundamentais que contemplam conteúdos das ciências biológicas, humanas, sociais, da saúde e da enfermagem. A prática clínica desenvolvida pelos estudantes inclui o cuidado da adolescente e da mulher adulta, antes, durante e depois da gravidez, nos mais diversos cenários de prestação de assistência em saúde, desde os comunitários até os hospitalares, especialmente os localizados na Zona Leste de São Paulo, região com a qual o Curso de Obstetrícia tem buscado parcerias para o desenvolvimento não só do ensino, como também para trabalhos de pesquisa e extensão. Os propósitos da pesquisa e extensão são aprofundar ou expandir temáticas para o desenvolvimento da ciência, da saúde e da assistência à saúde materno-infantil, bem como integrar o ensino com a pesquisa, em processo de retroalimentação que fortalece o aprendizado.

\section{As Resistências, os Desafios e as Perspectivas do Curso de Obstetrícia}

Por se tratar de um curso inovador e de formação desconhecida pela maioria população, os egressos do Curso de Obstetrícia vêm encontrando dificuldades de inserção no mercado de trabalho. Primeiramente, por ainda não dispor de um conselho que efetue inscrição e conceda registro profissional de forma regular.

Sobre esse aspecto ressalta-se que, de início, considerou-se que o sistema COFEN/COREN, que fiscaliza e disciplina o exercício de Enfermagem, poderia abrigar estes profissionais como já havia feito no passado. Afinal, algumas das obstetrizes formadas até a década de 70 continuam atuando na área, sendo devidamente registradas nos conselhos de enfermagem. Mas, a realidade tem mostrado que talvez não seja esse o caminho para os egressos do Curso de Obstetrícia da EACH-USP. A opção pela criação de um conselho profissional próprio demanda articulações políticas e exige maior tempo para inserção de obstetrizes no mercado de trabalho. Por outro lado, por se tratar de uma nova carreira do sistema de saúde, alguns recém-formados não 
puderam ainda assumir cargos públicos, mesmo quando aprovados.

Estes fatores têm influenciado a diminuição pela procura do curso, que de 15 candidatos por vaga no vestibular de 2005 baixou para 5,6 em 2011, índice inferior aos demais cursos da USP, especialmente os mais tradicionais na área da saúde. Observase, nesse sentido, como é fundamental o trabalho de divulgação constante e efetivo da necessidade desse tipo de formação, o que se está pouco a pouco fazendo por meio das atividades de ensino, pesquisa e extensão junto aos serviços de saúde, aos profissionais de saúde e, especialmente, às mulheres, famílias e comunidade.

Outro fator dificultador para a atuação de egressos do curso é o modelo biomédico e hospitalocêntrico que vigora no sistema de saúde brasileiro. Poucas são as casas de parto existentes no país; as que existem são subutilizadas por serem pouco conhecidas pela população e desprestigiadas pelos profissionais e sistemas de saúde. Muito embora em maior número, o mesmo ocorre com os centros de parto normal intra-hospitalares.

Pelo tipo de formação que recebem, os profissionais formados pelo Curso de Obstetrícia têm maior afinidade com estes contextos menos tecnológicos. Este fato já é verificado no processo de formação, nos diferentes campos de prática utilizados para o ensino teórico-prático, especialmente ao processo de parto e nascimento na rede hospitalar. O usual tem sido encontrar-se grande dificuldade para colocar em prática o modelo assistencial centrado nas necessidades da mulher/família e nas intervenções baseadas em evidências científicas que fundamentam todo o ensino, conforme descrito anteriormente.

Ilustra essa situação os resultados do trabalho de Narchi (2011) realizado em 2006 nos seis hospitais públicos com leitos obstétricos da Zona Leste do município de São Paulo. A autora verificou que naquelas instituições 80 a $100 \%$ dos partos de baixo risco eram assistidos por enfermeiras obstetras, a maioria deles ainda utilizando práticas tradicionais e desaconselhadas pelas evidências científicas e, até, prejudiciais à saúde materna e perinatal, por motivos relacionados à formação ou à resistência institucional a tudo o que cerca o processo de humanização do parto.

São em algumas dessas instituições, bem como em outras semelhantes, que se desenvolve a prática clínica do Curso de Obstetrícia, nos quais docentes e discentes enfrentam, juntamente com as mulheres, imposições contrárias ao que é discutido e preconizado durante o ensino teórico. Impede-se, desse modo, a implementação de medidas preconizadas pelas evidências científicas, recomendadas e exigidas por dispositivos legais, pactos e programas governamentais, que os gestores dos serviços públicos não colocam em prática, por não estarem afinados com as políticas públicas ou por não serem devidamente cobrados pelos órgãos responsáveis, pela população e pelos próprios profissionais de saúde.

A despeito do exposto, o processo ensino-aprendizagem dos estudantes do Curso de Obstetrícia baseia-se em discutir, refletir criticamente e procurar reverter o máximo possível essas situações, de forma que haja qualidade assistencial. Dessa maneira, mesmo com todas as dificuldades inerentes às particularidades de cada instituição, tem-se preconizado que cada mulher assistida durante o processo de parto e nascimento tenha a oportunidade de conhecer e participar ativamente de outra forma de cuidado, mais digna e respeitosa. Além disso, o exercício do ensino teórico-prático conduzido no sistema público de saúde desenvolve-se de forma a criar real compromisso do estudante com a modificação ou transformação do cenário em que ocorre a atenção ao parto e nascimento no SUS.

Entendendo que a conduta adotada no cuidado à mulher no Brasil tem sido marcada por desafios que precisam ser superados e que, ao longo do tempo, certamente o serão, tem-se como perspectiva que obstetrizes ocupem seu lugar em nosso país e contribuam para a necessária redução dos índices de morbi-mortalidade materna e perinatal e das cesáreas, para a "desmedicalização" do parto e para que as mulheres sejam mais bem assistidas, fazendo valer seus direitos humanos, sexuais e reprodutivos.

Nessa perspectiva, pesquisa realizada em $2007 \mathrm{e}$ 2008 com discentes do Curso de Obstetrícia (Jesus, $\left.2008^{1}\right)$ mostrou que a maioria deles se encontra

1 JESUS, G.M. Expectativas de docentes e discentes do curso de graduação em obstetrícia. Relatório de Pesquisa. Programa Ensinar com Pesquisa. São Paulo: EACH-USP, 2008 (mimeo). 
preparada para lidar com esses desafios, esperando em curto prazo atuar em maternidades públicas ou privadas, em centros de parto intra-hospitalares, em casas de parto e no domicílio.

Infelizmente, essa não tem sido até o momento a realidade, pois, como já previam os docentes do curso na citada pesquisa ${ }^{2}$, a incompreensão do potencial inovador desta nova profissão, a falta de investimentos públicos e privados na contratação de maior número de enfermeiras obstetras e obstetrizes, o ineditismo da profissão, o desvalor generalizado da sociedade brasileira para o trabalho em saúde pelo não médico, entre outros fatores, constituem-se entraves para a absorção desses profissionais pelo mercado.

A formação de opinião pública e de identidade própria, resultante da necessária regulamentação da profissão no Brasil, poderá pouco a pouco reverter tal situação, sendo estes alguns dos principais desafios que se apresentam para os docentes, discentes e os já formados, todos empenhados nessa verdadeira batalha que se tornou a inclusão de mais um profissional no cenário da assistência à saúde em nosso país.

Como o Brasil é um país continental, de grande diversidade sócio-econômica e cultural, com regiões em que o sistema de saúde é precário e desprovido de profissionais capacitados, planeja-se para o futuro envolver os estudantes em trabalhos de extensão e pesquisa junto a comunidades em que a atenção ao processo de parto e nascimento ocorra fora do ambiente hospitalar. Nessa perspectiva, é importante destacar a importância do estabelecimento de trabalho junto às parteiras leigas ou tradicionais, pessoas desvalorizadas, frequentemente desprezadas pelos profissionais de saúde, que trabalham sem vínculo com o sistema formal de saúde brasileiro, às vezes isoladas e em condições precárias. Elas são tidas como pessoas atrasadas, sem higiene e ignorantes, e, no entanto, utilizam práticas essenciais ao cuidado centrado na mulher, como o suporte emocional, o respeito, a facilitação do processo fisiológico por meio de posições variadas para o parto, o estímulo ao vínculo e ao aleitamento materno, entre outras (Rattner e col., 2009; Rattner, 2009a, 2009b).
Finalmente, avalia-se que o exercício das competências essenciais em obstetrícia requer o fortalecimento da identidade profissional tanto de enfermeiras obstetras quanto de obstetrizes, o que não é prática corrente nos serviços de saúde de nosso país. Nesse sentido, citando Davis-Floyd (1999), espera-se que obstetrizes e enfermeiras obstetras não concentrem sua atenção em disputas exclusivistas e contraproducentes entre si, o que as enfraquecerá. Por terem interesses em comum, espera-se que estas duas profissões juntem harmonicamente seus esforços na disputa pelo devido reconhecimento, respeito e conseqüente transformação no panorama de atenção à saúde das mulheres brasileiras.

\section{Referências}

ALBUQUERQUE, V. S. et al. Currículos disciplinares na área de saúde: ensaio sobre saber e poder. Interface: Comunicação, Saúde, Educação, Botucatu, v. 13, n. 31, p. 261-272, 2009.

AYRES, J. R. C. M. Hermeneutics and humanization of the health practices. Ciência \& Saúde Coletiva, Rio de Janeiro, v. 10, n. 3, p. 549$560,2005$.

BARRET, T. Philosophical principles for problembased learning: Freire's concepts of personal development and social empowerment. In: LITTLE, P.; KANDLBINDER, P. (Ed). The power of problem-based learning experience, empowerment, evidence. Newcastle: PROBLARC, 2001. p. 9-18.

BARROWS, H. S. Problem based learning in medicine and beyond: a brief overview. In: WILKERSON, L.; GILJSELAERS, W. H. (Ed.). Bringing problem-based learning to higher education theory and practice. San Francisco: Jossey Bass, 1996. p. 3-11.

BRASIL. Ministério da Saúde. Secretaria de Políticas de Saúde. Programa de humanização do pré-natal e nascimento. Brasília, DF, 2000.

BRASIL. Ministério da Saúde. Parto, aborto e puerpério: assistência humanizada à mulher. Brasília, DF, 2001.

2 JESUS, G.M. Expectativas de docentes e discentes do curso de graduação em obstetrícia. Relatório de Pesquisa. Programa Ensinar com Pesquisa. São Paulo: EACH-USP, 2008 (mimeo). 
BRASIL. Ministério da Saúde. Pacto nacional pela redução da mortalidade materna e neonatal. Brasília, DF, 2004. Disponível em: <http://dtrzoo2. saude.gov.br/proesf/Site/Arquivos_pdf_word/pdf/ Pacto\%2oAprovado\%2ona\%2oTripartite.pdf >. Acesso em: 15 maio 2011.

BRASIL. Agência Nacional de Saúde Suplementar (ANS). O modelo de assistência obstétrica no setor de saúde suplementar no Brasil: cenários e perspectivas. Rio de Janeiro, 2008.

BRASIL. Ministério da Saúde. Saúde Brasil 2oog: uma análise da situação de saúde e da agenda nacional e internacional de prioridades em saúde. Brasília, DF, 2010.

CALDERON, I. M. P.; CECATTI, J. G.; VEGA, C. E. P. Intervenções benéficas no pré-natal para prevenção da mortalidade materna. Revista Brasileira de Ginecologia e Obstetrícia, Rio de Janeiro, v. 28, n. 5, p. 310-315, 2006.

CARVALHO, V. Qualidade na formação da enfermeira para atuação na área perinatal: considerações substantivas e adjetivas do perfil profissional. In: CONGRESSO BRASILEIRO DE ENFERMAGEM OBSTÉTRICA E NEONATAL, 2., 200o, Rio de Janeiro. Anais... Rio de Janeiro: Associação Brasileira de Obstetrizes e Enfermeiros Obstetras, 200o. p. 15-25.

CECATTI, J. G.; CALDERÓN, I. M. P. Intervenções benéficas durante o parto para a prevenção da mortalidade materna. Revista Brasileira de Ginecologia e Obstetrícia, Rio de Janeiro, v. 27, n. 6, p. 357-365, 2005 .

COREN-SP - CONSELHO REGIONAL DE ENFERMAGEM DE SÃO PAULO. Principais legislações para o exercício da enfermagem. São Paulo, 2009.

COSTA, A. M.; GUILHEM, D.; WALTER, M. I. M. T. Atendimento a gestantes no Sistema Único de Saúde. Revista de Saúde Pública, São Paulo, v. 39, n. 5, p. 768-774, 2005.

DAVIS-FLOYD, R. Some thoughts on bridging the gap between nurse and direct-entry midwives. Midwifery Today, Eugene, n. 49, Spring 1999. Disponível em: <http://midwiferytoday.com/ articles/gap.asp>. Acesso em: 15 maio 2011.
DI MARIO, S. et al. What is the effectiveness of antenatal care? (Suplement) Copenhagen. WHO Regional Office for Europe (Health Evidence Network Report), 2005. Disponível em: < http://www.euro.who.int/__data/assets/ pdf_file/ooo5/7466o/E87997.pdf> Acesso em: 15 maio 2011.

FURLLERTON, J. T.; THOMPSON, J. B. Examining the evidence for the International Confederation of Midwives' essential competencies for midwifery practice. Midwifery, London, v. 21, n. 1, p. 2-13, 2005.

HATEM, M. et al. Midwife-led vs. other models of care for childbearing women. Cochrane Database of Systematic Reviews 2008, Issue 4. Art. No.: CDoo4667. DOI: 10.1002/14651858.CDoo4667. pub2. Disponível em: <http://onlinelibrary.wiley. com/doi/10.1002/14651858.CDoo4667.pub2/pdf/ abstract> Acesso em: 19 junho 2012.

ICM - INTERNATIONAL CONFEDERATION OF MIDWIVES. Code of ethics for midwives. Hague, 2005. Disponível em: <http://www. internationalmidwives.org>. Acesso em: 19 junho 2012.

ICM - INTERNATIONAL CONFEDERATION OF MIDWIVES. Competencies. Hague, 2002. Disponível em: <http://www. internationalmidwives.org/Portals/5/ Documentation/Essential\%20 Compsenglish_2002-JF_2007\%FINAL.pdf >. Acesso em: 15 maio 2011.

ICM - INTERNATIONAL CONFEDERATION OF MIDWIVES. Philosophy and model of care. Hague, 2005. Disponível em: <http://www. internationalmidwives.org>. Acesso em: 15 maio 2011.

JORGE, D. R. Evolução da legislação federal do ensino e do exercício profissional da obstetriz (parteira) no Brasil. 1975. Tese (Livre Docência em Enfermagem) - Escola de Enfermagem Ana Neri da UFRJ, Rio de Janeiro, 1975.

LANSKY, S.; FRANÇA, E.; LEAL, M. C. Mortalidade perinatal e evitabilidade: revisão da literatura. Revista de Saúde Pública, São Paulo, v. 36, n. 6, p. 759-772, 2002. 
MAIA, J. A. Formação humanística no ensino superior em saúde: intencionalidades e acasos. In: BATISTA, N. A.; BATISTA, S. H.; ABDALLA, I. G. (Org.). Ensino em saúde: visitando conceitos e práticas. São Paulo: Arte \& Ciência, 2005. p. 39-54.

MC COURT, C.; THOMAS, B. G. Evaluation of a problem-based curriculum in midwifery. Midwifery, London, v. 17, n. 4, p. 323-331, 2001.

NARCHI, N. Z. Aspectos legais do ensino e exercício profissional da enfermeira obstétrica: transformações e perspectivas. In: SEMINÁRIO ESTADUAL SOBRE O ENSINO DE ENFERMAGEM PARA A ASSISTÊNCIA AO NASCIMENTO E PARTO, 3., 2001, São Paulo. Anais... São Paulo: Associação Brasileira de Obstetrizes e Enfermeiros Obstetras: Escola de Enfermagem da Universidade de São Paulo, 2001, p. 93-101.

NARCHI, N. Z. Exercise of essential competencies for midwifery care by nurses in São Paulo, Brazil. Midwifery, London, v. 27, n. 1, p. 23-29, 2011.

OEA - ORGANIZACIÓN DE LOS ESTADOS AMERICANOS. Acceso a servicios de salud materna desde una perspectiva de derechos humanos. Washington, DC, 2006.

PINHEIRO, R.; MATTOS, R.; CAMARGO Jr., R. M. K. R. (Org.). Construção da integralidade: cotidiano, saberes e práticas em saúde. Rio de Janeiro: UERJ: IMS Abrasco, 2003.

RATTNER, D. Humanização na atenção a nascimentos e partos: breve referencial teórico. Interface: Comunicação, Saúde, Educação, Botucaru, v. 13, p. 595-602, 2009a. Suplemento 1.

RATTNER, D. Humanização na atenção a nascimentos e partos: ponderações sobre políticas públicas. Interface: Comunicação, Saúde, Educação, Botucatu, v. 13, p. 759-768, 20ogb.

Suplemento 1.
RATTNER, D. et al. Humanizing childbirth to reduce maternal and neonatal mortality: a national effort in Brazil. In: DAVIS-FLOYD, R. et al. (Org.). Birth models that work. Berkeley: University of California Press, 2009. p. 385-413.

ROWAN, C.; MC COURT, C.; BEAKE, S.

Midwives'reflections on their educational programme: a traditional or problem-based learning approach? Midwifery, London, v. 25, n. 2, p. 213-222, 2009.

UNFPA - UNITED NATIONS POPULATION FOUND. Towards MDG 5: scaling up the capacity of midwives to reduce maternal mortality and morbidity. New York, 2006.

UNFPA - UNITED NATIONS POPULATION FOUND. Maternal mortality update 20o6: expectation and delivery: investing in midwives and others with midwifery skills. New York, 2007. Disponível em: < http://www.unfpa.org/webdav/site/global/shared/ documents/publications/2007/mm_updateo6_eng. pdf >. Acesso em: 15 maio 2011.

WHO - WORLD HEALTH ORGANIZATION. Safe motherhood needs assessment: guidelines.

Geneva, 2001. Disponível em: <http://www.who.int/ reproductivehealth/MNBH/smna_index.en.html $>$. Acesso em: 15 maio 2011.

WHO - WORLD HEALTH ORGANIZATION. Department of Making Pregnancy Safer. Strengthening midwifery toolkit: developing a midwifery curriculum for safe motherhood. Geneva, 2006.

WHO - WORLD HEALTH ORGANIZATION. Department of Reproductive Health and Research. Making pregnancy safer: the critical role of the skilled attendant. Geneva, 2004.

Recebido em: 17/05/2010

Reapresentado em: 11/01/2012

Aprovado em: 17/03/2012 